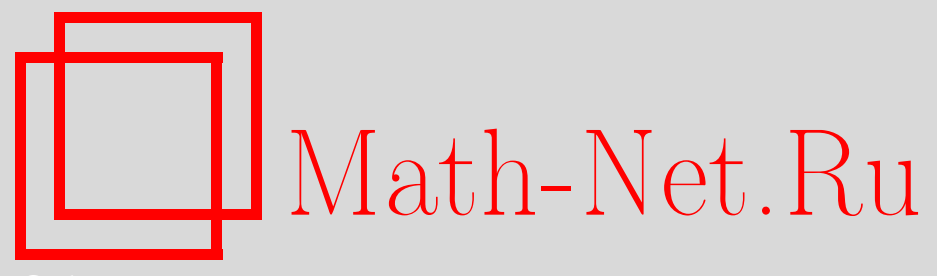

Е. Е. Дьяконова, Об асимптотике вероятности невырождения многомерного ветвящегося процесса в случайной среде, Дискрет. матем., 1999, том 11, выпуск 1, 113-128

DOI: https://doi.org/10.4213/dm361

Использование Общероссийского математического портала Math-Net.Ru подразумевает, что вы прочитали и согласны с пользовательским соглашением http://www. mathnet.ru/rus/agreement

Параметры загрузки:

IP: 34.239 .49 .27

26 апреля 2023 г., 09:06:20

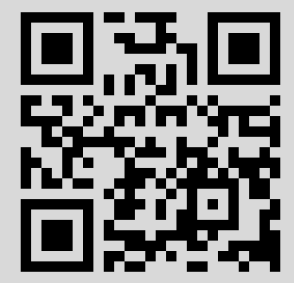




\title{
Об асимптотике вероятности невырождения многомерного ветвящегося процесса в случайной среде
}

\author{
(c) 1999 г. $\quad$ Е. Е. Дьяконова
}

\begin{abstract}
Рассматривается многомерный ветвящийся процесс Гальтона-Ватсона в случайной среде, порожденной последовательностью независимых одинаково распределенных случайных величин. Показано, что асимптотика вероятности невырождения этого процесса в момент времени $n$ при некоторых условиях, наложенных на производящие функции числа потомков, имеет порядок $n^{-1 / 2}$ при $n \rightarrow \infty$.

Работа выполнена при поддержке Российского фонда фундаментальных исследований, проекты 96-01-00338 и 96-15-96092, и INTAS-RFBR, проект 950099 .
\end{abstract}

\section{1. Введение}

Изучению поведения ветвящихся процессов в случайной среде посвящено большое количество работ (см., например, $[1,2,4-10])$. Впервые модель ветвящегося процесса в случайной среде была рассмотрена Смитом и Вилкинсоном [1]. В последующих работах [2, 5-7] наряду с одномерными процессами исследовались и многомерные процессы Гальтона-Ватсона в случайной среде. Асимптотика вероятности невырождения одномерного критического ветвящегося процесса в случайной среде была изучена в [9]. В настоящей работе результаты, полученные в [9], обобщаются на случай многомерных ветвящихся процессов.

Мы будем рассматривать ветвящийся процесс Гальтона-Ватсона

$$
Z(n)=\left(Z_{1}(n), \ldots, Z_{p}(n)\right), \quad n=0,1, \ldots,
$$

c $p>1$ типами частиц в случайной среде $\zeta$, который может быть определен следующим образом. Пусть $\zeta=\left\{\zeta_{n}, n=0,1, \ldots\right\}$ - последовательность независимых одинаково распределенных случайных величин, определенных на вероятностном пространстве $(\Omega, \mathscr{F}, \mathbf{P})$ и принимающих значения из счетного подмножества $\Theta$ множества действительных чисел.

В дальнейшем будем обозначать $\alpha=\left(\alpha_{1}, \ldots, \alpha_{p}\right)^{T}$ и $\widetilde{\alpha}=\left(\alpha_{1}, \ldots, \alpha_{p}\right)$, соответственно, вектор-столбец и вектор-строку с координатами $\alpha_{1}, \ldots, \alpha_{p}$. Пусть $N^{p}$ - множество всех векторов $\widetilde{\alpha}=\left(\alpha_{1}, \ldots, \alpha_{p}\right)$ с целочисленными неотрицательными координатами. Предположим, что каждой точке $\theta \in \Theta$ поставлен в соответствие 
$p$-мерный вектор-столбец $F^{(\theta)}(s)=\left(F_{1}^{(\theta)}(s), \ldots, F_{p}^{(\theta)}(s)\right)^{T}$ вероятностных производящих функций

$$
\begin{gathered}
F_{i}^{(\theta)}(s)=\sum_{\widetilde{\alpha} \in N^{p}} b(i, \theta, \widetilde{\alpha}) \prod_{i=1}^{p}\left(s_{i}\right)^{\alpha_{i}}, \quad s=\left(s_{1}, \ldots, s_{p}\right)^{T}, \\
0 \leqslant s_{i} \leqslant 1, \quad i=1, \ldots, p, \quad \cdot \widetilde{\alpha}=\left(\alpha_{1}, \ldots, \alpha_{p}\right) .
\end{gathered}
$$

Процесс Гальтона-Ватсона с $p$ типами частиц в случайной среде $\zeta$ описывает эволюцию популяции частиц $Z(n)=\left(Z_{1}(n), \ldots, Z_{p}(n)\right), n=0,1, \ldots$, где $Z_{i}(n)$, $i=1, \ldots, p,-$ число частиц типа $i$, присутствующих в процессе в момент времени $n$. Предполагается, что в если $\zeta_{n}=\theta, \theta \in \Theta$, то в момент времени $n$ все $Z_{i}(n)$ частиц $i$-го, $i=1, \ldots, p$, типа размножаются согласно $p$-мерной производящей функции $F_{i}^{(\theta)}(s)$, причем количество потомков любой частицы не зависит от числа потомков других частиц данного типа и от числа потомков частиц других типов. Таким образом, компонента $Z_{i}(n+1), i=1, \ldots, p$, вектора $Z(n+1)$ равняется числу частиц $i$-го типа среди всех потомков частиц $n$-го поколения $Z(n)$, при этом $b(i, \theta, \widetilde{\alpha})$ есть вероятность того, что частица $i$ из $n$-го поколения при условии $\zeta_{n}=\theta$ будет иметь $\alpha_{j}$ потомков типа $j, j=1, \ldots, p, \widetilde{\alpha}=\left(\alpha_{1}, \ldots, \alpha_{j}\right)$.

Введем ряд обозначений. Пусть $I$ - единичный, 0 - нулевой $p$-мерные векторыстолбцы, $\widetilde{e}_{j}-p$-мерный вектор-строка, $j$-я компонента которого равняется единице, а все остальные нулю. Для $\widetilde{x}=\left(x_{1}, \ldots, x_{p}\right), y=\left(y_{1}, \ldots, y_{p}\right)^{T}$ будем обозначать

$$
|\widetilde{x}|=\sum_{i=1}^{p}\left|x_{i}\right|, \quad|y|=\sum_{i=1}^{p}\left|y_{i}\right|, \quad \tilde{x} y=\sum_{i=1}^{p} x_{i} y_{i} .
$$

Пусть $A=|A(i, j)|_{i, j=1}^{p}-$ произвольная положительная матрица размера $p \times p$. Положим

$$
\|A\|=\max _{i=1, \ldots, p} \sum_{j=1}^{p} A(i, j) .
$$

Обозначим $\rho(A)$ - перронов корень матрицы $A$. Пусть $r(A)=\left(r_{1}(A), \ldots, r_{p}(A)\right)^{T}$ и $\tilde{l}(A)=\left(l_{1}(A), \ldots, l_{p}(A)\right)$ - правый и, соответственно, левый собственные векторы матрицы $A$, соответствующие собственному значению $\rho(A)$ и удовлетворяющие условиям нормировки

$$
\widetilde{l}(A) r(A)=1, \quad|\widetilde{l}(A)|=1
$$

Нам понадобится также еще одна пара векторов, состоящая из правого $R(A)=$ $\left(R_{1}(A), \ldots, R_{p}(A)\right)^{T}$ и левого $\widetilde{L}(A)=\left(L_{1}(A), \ldots, L_{p}(A)\right)$ собственных векторов матрицы $A$, соответствующих собственному значению $\rho(A)$ и нормированных так, что

$$
\widetilde{L}(A) R(A)=1, \quad|\widetilde{R}(A)|=1 .
$$

Пусть, далее,

$$
M_{\zeta_{\mathfrak{n}}}=\left|M_{\zeta_{n}}(i, j)\right|_{i, j=1}^{p}=\left|\frac{\partial F_{i}^{\left(\zeta_{n}\right)}(I)}{\partial s_{j}}\right|_{i, j=1}^{p}
$$


- случайная матрица математических ожиданий векторной (случайной) производящей функции $F^{\left(\zeta_{n}\right)}(s), M^{(\theta)}=\left|M^{(\theta)}(i, j)\right|_{i, j=1}^{p}=M_{\zeta_{n}}$ при $\zeta_{n}=\theta, \theta \in \Theta, \rho_{\zeta_{n}}=\rho\left(M_{\zeta_{n}}\right)$, $n=0,1, \ldots, \rho^{(\theta)}=\rho\left(M^{(\theta)}\right)$.

Введем также матрицы

$$
\begin{gathered}
B_{\zeta_{n}}^{(k)}=\left|B_{\zeta_{n}}^{(k)}(i, j)\right|_{i, j=1}^{p}=\left|\frac{\partial^{2} F_{k}^{\left(\zeta_{n}\right)}(I)}{\partial s_{i} \partial s_{j}}\right|_{i, j=1}^{p}, \quad k=1, \ldots, p, \\
B^{(\theta, k)}=\left|B^{(\theta, k)}(i, j)\right|_{i, j=1}^{p}=B_{\zeta_{n}}^{(k)}, \quad \zeta_{n}=\theta, \quad \theta \in \Theta, \quad n=0,1, \ldots
\end{gathered}
$$

В дальнейшем, там где это не вызывает недоразумений, вместо индекса $\zeta_{n}$ будем писать $n$, например, $M_{n}=\left|M_{n}(i, j)\right|_{i, j=1}^{p}, B_{n}^{(k)}=\left|B_{n}^{(k)}(i, j)\right|_{i, j=1}^{p}, \rho_{n}$ вместо $M_{\zeta_{n}}=$ $\left|M_{\zeta_{n}}(i, j)\right|_{i, j=1}^{p}, B_{\zeta_{n}}^{(k)}=\left|B_{\zeta_{n}}^{(k)}(i, j)\right|_{i, j=1}^{p}, \rho_{\zeta_{n}}$. Заметим, что $\rho_{n}, n=0,1, \ldots$, образуют последовательность независимых одинаково распределенных случайных величин.

$\mathrm{C}$ каждым $i=1, \ldots, p$ свяжем случайный момент $T_{i}$ вырождения процесса $Z(n)$ при условии, что $Z(0)=\widetilde{e}_{i}$ :

$$
T_{i}=n \Longleftrightarrow Z(0)=\widetilde{e}_{i}, \quad Z(n-1) \neq \widetilde{0}, \quad Z(n)=\widetilde{0}, \quad \widetilde{0}=(0, \ldots, 0) .
$$

Известно [7], что

$$
\mathbf{P}\left(T_{i}>n\right)=\mathbf{M} \widetilde{e}_{i}\left(I-F^{\left(\zeta_{0}\right)}\left(F^{\left(\zeta_{1}\right)} \ldots\left(F^{\left(\zeta_{n-1}\right)}(0)\right)\right)\right) .
$$

В работах $[2,7]$ показано, что для неразложимого ветвящегося процесса $Z(n)$, имеющего ограниченные первые и вторые моменты и функционирующего в случайной среде $\zeta$, существует с вероятностью единица предел

$$
\lim _{n \rightarrow \infty} \frac{1}{n} \log \left\|\prod_{i=0}^{n-1} M_{i}\right\|=\pi,
$$

причем

$$
\lim _{n \rightarrow \infty} \frac{1}{n} \mathbf{M} \log \left\|\prod_{i=0}^{n-1} M_{i}\right\|=\pi .
$$

Известно $[2,7]$, что если $\pi \leqslant 0$, то процесс $Z(n)$ вырождается с вероятностью единица.

\section{2. Формулировка основных результатов}

Предположим, что существуют такие постоянные $m>0, b>0,1<D_{1}<D_{2}<\infty$, что при всех значениях $\theta \in \Theta, i, j, k=1, \ldots, p$,

$$
\begin{aligned}
& m \leqslant M^{(\theta)}(i, j) \leqslant D_{1}, \\
& b \leqslant B^{(\theta, k)}(i, j) \leqslant D_{2} .
\end{aligned}
$$

Кроме того, предположим, что выполняется следующее условие $A$ : существует такой положительный вектор $r=\left(r_{1}, \ldots, r_{p}\right)^{T}$, что при любом $\theta \in \Theta$

$$
r\left(M^{(\theta)}\right)=r .
$$


Таким образом, все матрицы $M^{(\theta)}, \theta \in \Theta$, математических ожиданий векторных производящих функций $F^{(\theta)}(s), s=\left(s_{1}, \ldots, s_{p}\right)^{T}$, имеют общий положительный правый собственный вектор $r$ :

$$
M^{(\theta)} r=\rho\left(M^{(\theta)}\right) r
$$

Теорема 1. Предположим, что для ветвящегося процесса $Z(n)$ в случайной среде $\zeta$ выполнены условия $A,(5),(6) u$

$$
\mathbf{M} \ln \rho_{0}=0, \quad 0<\mathbf{M}\left(\ln \rho_{0}\right)^{2}<\infty .
$$

Тогда при некоторых постоянных $0<c_{1}<c_{2}<\infty$ справедливы неравенства

$$
c_{1} / \sqrt{n} \leqslant \mathbf{P}\left(T_{i}>n\right) \leqslant c_{2} / \sqrt{n}, \quad n \geqslant 1, \quad i=1, \ldots, p .
$$

Введем класс $H\left(m, D_{1}, D_{2}\right)$ дробно-линейных многомерных производящих функций $F^{(\theta)}(s), \theta \in \Theta$, состоящий из $F^{(\theta)}(s)$, удовлетворяющих условиям $A,(5),(6)$ и имеющих вид

$$
\begin{gathered}
F^{(\theta)}(s)=I-\frac{M^{(\theta)}(I-s)}{1+\widetilde{\gamma}(\theta)(I-s)}, \quad \theta \in \Theta, \\
\widetilde{\gamma}(\theta)=\left(\gamma_{1}(\theta), \ldots, \gamma_{p}(\theta)\right), \quad \gamma_{j}(\theta)=\gamma^{(\theta)}, \quad j=1, \ldots, p, \\
\frac{D_{2}}{2 D_{1}} \geqslant \gamma^{(\theta)} \geqslant \frac{p D_{1}-1}{K}, \quad D_{2} \geqslant \frac{2 p D_{1}^{2}}{K}, \\
K=\min \{p,|r|\}, \quad b=\frac{2 m}{K}\left(p D_{1}-1\right) .
\end{gathered}
$$

Теорема 2. При выполнении условий теоремъ 1 для ветвящихся процессов $Z(n)$ в случайной среде $\zeta$, имеющих дробно-линейные многомернъе производящие функции $F^{(\theta)}(s) \in H\left(m, D_{1}, D_{2}\right)$, при $n \rightarrow \infty$ имеет место соотношение

$$
\mathbf{P}\left(T_{i}>n\right) \sim \frac{d_{i}}{\sqrt{n}}, \quad d_{i}>0 .
$$

Заметим, что из (12) следует, что

$$
F_{i}^{(\theta)}(0) \geqslant 1-\frac{\left\|M^{(\theta)}\right\|}{1+\gamma^{(\theta)} p} \geqslant 0, \quad i=1, \ldots, p,
$$

то есть соотношение (11) действительно определяет многомерную производящую функцию случайного вектора.

Несложно показать, что выполняется следующее утверждение.

Следствие. При замене в формулировках теорем 1, 2 условия $A$ на условие $A^{\prime}$, состоящее в том, что все матрицы $M^{(\theta)}, \theta \in \Theta$, имеют общий положителъный левый собственный вектор $\widetilde{L}=\widetilde{L}\left(M^{(\theta)}\right)$, утверждения теорем 1,2 остаются в cuлe.

Отметим, что ключевым моментом в доказательстве теорем 1,2 является сходимость по распределению произведения $\prod_{i=1}^{n} M_{i} / \rho_{i}$ к ненулевой матрице при $n \rightarrow \infty$.

Известно [14], что в случае $p=2$ произведение $\prod_{i=1}^{n} M_{i} / \rho_{i}$ положительных ограниченных матриц $A_{i}=M_{i} / \rho_{i}$ сходится по распределению к ненулевой матрице при $n \rightarrow \infty$ тогда и только тогда, когда матрицы $A_{i}$ имеют общие положительные правые или левые собственные векторы, поэтому в случае двумерного процесса $Z(n)$ невозможно отказаться от требования наличия общего положительного правого или левого собственного вектора у матриц $M^{(\theta)}, \theta \in \Theta$. 


\section{3. Доказательства теорем}

Докажем сначала теорему 2. Обозначим

$$
q^{i}(n)=\mathbf{P}\left(T_{i}>n\right) .
$$

Введем случайную матрицу

$$
E_{k, n}=M_{k} \ldots M_{n}, \quad k \leqslant n .
$$

Из условия (5) следует, что для всех $\theta \in \Theta$, всех $n \geqslant 0$ и всех $i_{1}, i_{2}, j_{1}, j_{2}$

$$
a \leqslant \frac{M_{n}\left(i_{1}, j_{1}\right)}{M_{n}\left(i_{2}, j_{2}\right)} \leqslant a^{-1}
$$

где

$$
0<a \leqslant \frac{m}{D_{1}}<1 .
$$

Известно ([15], лемма 1), что для произведения $E_{k, n}=\left|E_{k, n}(i, j)\right|_{i, j=1}^{p}$ матриц $M_{k}, \ldots, M_{n}$, удовлетворяющих условиям $A,(14)$, справедливо представление

$$
E_{k, n}(i, j)=\prod_{v=k}^{n} \rho_{v} r_{i} l_{j}\left(E_{k, n}\right)\left(1+\mu_{k, n}(i, j) \beta^{n-k+1}\right)
$$

где

$$
\beta=\frac{1-a^{2}}{1+a^{2}}, \quad\left|\mu_{k, n}(i, j)\right| \leqslant C_{1}=C_{1}(a),
$$

где $C_{1}$ - постоянная, $r=\left(r_{1}, \ldots, r_{p}\right)$ - собственный вектор из условия $A$.

Суммируя $E_{k, n}(i, j)$ по $j$ от 1 до $p$ и учитывая соотношения (2), из (15) получаем, что

$$
\left|\frac{\widetilde{e}_{i} \prod_{j=k}^{n} M_{j} I}{\prod_{j=k}^{n} \rho_{j}}-r_{i}\right| \leqslant C_{1} \beta^{n-k+1}, \quad i=1, \ldots, p,
$$

где $C_{1}$ - постоянная. Заметим, что из (9) и (17) следует, что для процесса $Z(n)$, удовлетворяющего условиям теоремы 1 ,

$$
\lim _{n \rightarrow \infty} \frac{1}{n} \log \left\|\prod_{i=0}^{n} M_{i}\right\|=0 \text { (п.н.), }
$$

то есть (см., например, [7]) $Z(n)$ вырождается с вероятностью единица.

Будем обозначать $\widetilde{\gamma}_{n}=\widetilde{\gamma}\left(\zeta_{n}\right), F_{n}(s)=F^{\left(\zeta_{n}\right)}(s), \gamma_{(n)}=\gamma^{\left(\zeta_{n}\right)}, n=0,1, \ldots$, (cp. с (11), (12)).

Лемма 1. В условиях теоремы 2

$$
q^{i}(n)=\mathbf{M} \frac{\widetilde{e}_{i} M_{0} \ldots M_{n-1} I}{1+\widetilde{\gamma}_{0} M_{1} \ldots M_{n-1} I+\widetilde{\gamma}_{1} M_{2} \ldots M_{n-1} I+\ldots+\widetilde{\gamma}_{n-1} I} .
$$


Доказателъство. Применяя интегрирование, несложно получить, что для дробнолинейных производящих функций $F^{(\theta)}(s)$ имеет место соотношение

$$
\begin{aligned}
I-F_{0}\left(F_{1} \ldots\left(F_{n-1}(s)\right) \ldots\right) \\
=\frac{M_{0}\left(I-F_{1}\left(\ldots\left(F_{n-1}(s)\right) \ldots\right)\right)}{1+\widetilde{\gamma}_{0}\left(I-F_{1}\left(\ldots\left(F_{n-1}(s)\right) \ldots\right)\right)} \\
=\frac{M_{0} M_{1}\left(I-F_{2}\left(\ldots\left(F_{n-1}(s)\right) \ldots\right)\right)}{1+\widetilde{\gamma}_{0} M_{1}\left(I-F_{2}\left(\ldots\left(F_{n-1}(s)\right) \ldots\right)\right)+\widetilde{\gamma}_{1}\left(I-F_{2}\left(\ldots\left(F_{n-1}(s)\right) \ldots\right)\right)} \\
=\frac{M_{0} \ldots M_{n-1}(I-s)}{1+\widetilde{\gamma}_{0} M_{1} \ldots M_{n-1}(I-s)+\widetilde{\gamma}_{1} M_{2} \ldots M_{n-1}(I-s)+\ldots+\widetilde{\gamma}_{n-1}(I-s)} .
\end{aligned}
$$

Полагая в (20) $s=0$ и используя представление (3), получаем утверждение леммы.

Несложно видеть, что в условиях теоремы 2

$$
\frac{\partial^{2} F_{l}^{(\theta)}(I)}{\partial s_{i} \partial s_{j}}=\gamma^{(\theta)}\left(M^{(\theta)}(l, j)+M^{(\theta)}(l, i)\right), \quad l, i, j=1, \ldots, p .
$$

Из $(5),(6),(21)$ следует, что существуют такие постоянные $a_{1}, a_{2}, 0<a_{1}<a_{2}$, что

$$
a_{1}<\gamma_{(n)}<a_{2}, \quad n=0,1, \ldots
$$

Из (17), (19) следует, что

$$
q^{i}(n)=\mathbf{M} \frac{\widetilde{e}_{i} \prod_{i=0}^{n-1} \rho_{i}(r+t(n-1,0))}{1+\widetilde{\gamma}_{0} \prod_{i=1}^{n-1} \rho_{i}(r+t(n-1,1))+\widetilde{\gamma}_{1} \prod_{i=2}^{n-1} \rho_{i}(r+t(n-1,2))+\ldots+\widetilde{\gamma}_{n-1} I},
$$

где

$$
t(n, k)=\prod_{i=k}^{n} \frac{M_{i}}{\rho_{i}} I-r, \quad t(n, k)=\left(t_{1}(n, k), \ldots, t_{p}(n, k)\right), \quad k=1, \ldots, n-1,
$$

причем для $t_{j}(n, k), j=1, \ldots, p$, выполняется неравенство

$$
\left|t_{j}(n, k)\right| \leqslant C_{1} \beta^{n-k+1},
$$

где $C_{1}-$ положительная постоянная, $0<\beta<1$.

Заметим, что

$$
0<r_{j}+t_{j}(n, k), \quad j=1, \ldots, p .
$$

Пусть

$$
\begin{array}{rlrl}
X_{i} & =\ln \rho_{i-1}, & & \\
\widetilde{\mu}_{i} & =\frac{\widetilde{\gamma}_{i-1}}{\rho_{i-1}}=\left(\mu_{i}, \ldots, \mu_{i}\right), & \mu_{i}=\frac{\gamma_{(i-1)}}{\rho_{i-1}} \\
\eta_{i} & =\mu_{i} \sum_{j=1}^{p} r_{j}, & i & =1,2, \ldots
\end{array}
$$


Несложно видеть, что $\eta_{i}>0, i=1,2, \ldots$, a $\left(X_{i}, \eta_{i}\right)_{i=1}^{\infty}$ есть последовательность независимых одинаково распределенных двумерных случайных векторов, причем, как это следует из (9), (22), (5);

$$
\mathbf{M} \eta_{1}<\infty, \quad \mathbf{M}\left|\eta_{1} X_{1}\right|<\infty, \quad 0<\mathbf{M} X_{1}^{2}<\infty
$$

так как в силу (22) и (5) найдется такая постоянная $C>0$, что

$$
\eta_{i} \in(0, C], \quad i=1,2, \ldots
$$

Рассмотрим случайное блуждание $S_{n}=X_{1}+\ldots+X_{n}, S_{0}=0$, порожденное последовательностью $\left\{X_{i}, i=1,2, \ldots\right\}$ независимых одинаково распределенных случайных величин $X_{i}=\ln \rho_{i-1}, i=1,2, \ldots$ Введем строгие нижние лестничные моменты $\tau_{j}, j=0,1,2, \ldots$, полагая $\tau_{0}=0$ и $\tau_{j}=n$, если $\tau_{j-1}<n, S_{i} \geqslant S_{\tau_{j-1}}$ при $i<n$, $S_{n}<S_{\tau_{j-1}}$.

Пусть символ $\mathbf{M}_{n}$ означает математическое ожидание при условии $\tau_{1}>n$.

Из (23) следует, что

$$
\begin{aligned}
q^{i}(n) & =\mathbf{M}\left\{\frac{\tilde{e}_{i} r+\tilde{e}_{i} t(n-1,0)}{\prod_{i=0}^{n-1} \rho_{i}^{-1}+\sum_{i=0}^{n-1} \prod_{j=0}^{i-1} \rho_{j}^{-1} \tilde{\mu}_{i+1}(r+t(n-1, i))}\right\} \\
& =\mathbf{M}\left\{\frac{r_{i}+t_{i}(n-1,0)}{e^{-S_{n}}+\sum_{i=0}^{n-1} e^{-S_{i}} \eta_{i+1}(1+d(n-1, i))}\right\}, \quad i=1, \ldots, p,
\end{aligned}
$$

где случайные величины

$$
d(n-1, i)=\frac{1}{|r|} \sum_{j=1}^{p} t_{j}(n-1, i)>-1
$$

в силу (25).

Кроме того, из (5), (7) и (24) следует, что

$$
|d(n, k)| \leqslant \varphi(n-k+1), \quad k \leqslant n,
$$

где

$$
\varphi(n)=C_{1} \beta^{n}, \quad C_{1}>0, \quad 0<\beta<1,
$$

$C_{1}$ и $\beta$ - постоянные. Легко также видеть, что распределение случайной величины $d(n+k, i+k), i \leqslant n, k \geqslant 0$, не зависит от $k$.

Положим

$$
h(n)=\left(e^{-S_{n}}+\sum_{i=0}^{n-1} e^{-S_{i}} \eta_{i+1}(1+d(n-1, i))\right)^{-1}
$$

Исследуем асимптотику $\mathrm{M} h(n)$ при $n \rightarrow \infty$. Введем в рассмотрение одномерный процесс Гальтона-Ватсона $W(n), n=0,1, \ldots$, в нашей случайной среде $\zeta$ со следующими дробно-линейными производящими функциями:

$$
f_{i-1}(z)=1-\frac{e^{X_{i}}(1-z)}{1+e^{X_{i}} \eta_{i}(1-z)} .
$$


Отметим, что из (12) следует, что

$$
f_{i}(0)=1-\frac{\rho_{i}}{1+\gamma_{(i)} K} \geqslant 0, \quad K=\min \{p,|r|\}
$$

В силу определения случайных величин $\eta_{i}, X_{i}, i=1,2, \ldots$,

$$
\eta_{i}>0, \quad \mathbf{M} X_{i}=0, \quad 0<\mathbf{M} X_{i}^{2}<\infty, \quad \mathbf{M} \eta_{i}<\infty, \quad \mathbf{M}\left|\eta_{i} X_{i}\right|<\infty .
$$

Процесс $W(n), n=0,1, \ldots$, изучался М. В. Козловым в работе [9], в которой была получена асимптотика вероятности невырождения процесса $W(n)$ на основе исследования поведения $\mathrm{M} Y(n), n \rightarrow \infty$, где

$$
Y(n)=\left(e^{-S_{n}}+\sum_{i=0}^{n-1} e^{-S_{i}} \eta_{i+1}\right)^{-1}
$$

При этом существенно использовался тот факт, что $\left(X_{i}, \eta_{i}\right), i=1,2, \ldots$, являются независимыми одинаково распределенными случайными векторами. Для пар $\left(X_{i}, \eta_{i}+\eta_{i} d(n-1, i)\right), i=1,2, \ldots$, это не так, поскольку случайная величина $d(n-1, i)$ зависит от $X_{i}, \ldots, X_{n-1}$.

Пусть $N$ - множество натуральных чисел, $\Rightarrow-$ знак сходимости по распределению. Положим

$$
\begin{aligned}
Y(n, a, b)= & \left(a+b\left(e^{-S_{n}}+\sum_{i=0}^{n-1} \eta_{i+1} e^{-S_{i}}\right)\right)^{-1}, \quad a \geqslant 0, \quad b \geqslant 1, \\
h^{+}(n, a, b, v, l, c)= & (a(1-\varphi([v n])) \\
& \left.+b(1+c \varphi([\ln ]))^{-1}\left(\sum_{i=0}^{n-1} \eta_{i+1} e^{-S_{i}}(1+d(n-1, i))+e^{-S_{n}}\right)\right)^{-1}, \\
h_{-}(n, a, b, v, l, c)= & (a(1+\varphi([v n])) \\
& \left.+b(1+c \varphi([\ln ]))\left(\sum_{i=0}^{n-1} \eta_{i+1} e^{-S_{i}}(1+d(n-1, i))+e^{-S_{n}}\right)\right)^{-1},
\end{aligned}
$$

$a \geqslant 0, b \geqslant 1, c>0, v>0, l>0$.

Разобьем область интегрирования в $\mathrm{M} h(n)$ на части $\left(\tau_{j} \leqslant n, \tau_{j+1}>n\right), j=$ $0,1, \ldots, m-1$, и $\left(\tau_{m} \leqslant n\right)$. Известно ([9], формулы $\left.(16),(17)\right)$, что при выполнении условий (34), при $n \rightarrow \infty$,

$$
\mathbf{P}\left(\tau_{1}>n\right) \sim c_{3} / \sqrt{n}, \quad \mathbf{P}\left(\tau_{j} \geqslant n, \tau_{j+1}>n\right) \sim c_{3} / \sqrt{n}
$$

где $c_{3}$ - положительная постоянная. Кроме того, в [9], теорема В, показано, что при $n \rightarrow \infty$

$$
\left\{S_{i}, \eta_{i}, i \in N \mid \tau_{1}>n\right\} \Rightarrow\left\{\widehat{S}_{i}, \widehat{\eta}_{i}, i \in N\right\}
$$


где $\left\{\widehat{S}_{i}\right\}$ - некоторая однородная марковская цепь, выходящая из нуля, принимающая неотрицательные значения и не сосредоточенная в нуле, а случайные величины $\widehat{\eta}_{i}, i \in N$, неотрицательны с вероятностью единица. Следуя работе [9], результаты которой будут существенно использоваться в дальнейшем, сохраним для удобства изложения обозначение $\left\{S_{n}, \eta_{n}\right\}$ для процесса с конечномерными распределениями, задаваемыми правой частью (37), но в случае, когда речь идет об этом процессе, будем использовать для вероятности и математического ожидания обозначения $\widehat{\mathbf{P}}$ и $\widehat{M}$, соответственно.

Лемма 2. При $n \rightarrow \infty$

$$
\mathrm{M}\left(h(n) ; \tau_{1}>n\right) \sim c_{3} A_{0} / \sqrt{n}
$$

где $c_{3}-$ постоянная из выражсения (36),

$$
A_{0}=\widehat{\mathrm{M}}\left(\sum_{i=0}^{\infty} \eta_{i+1} e^{-S_{i}}\right)^{-1}, \quad A_{0} \in(0, \infty)
$$

Доказателъство. В ([9], стр.819-821) показано, что при $n \rightarrow \infty$

$$
\mathbf{M}\left(Y(n) ; \tau_{1}>n\right) \sim c_{3} A_{0} / \sqrt{n}
$$

и $0<A_{0}<\infty$. Положим $V_{i}(n)=1-\widetilde{e}_{i} F_{0}\left(F_{1} \ldots\left(F_{n-1}(0)\right) \ldots\right)$, то есть

$$
V_{i}(n)=\frac{r_{i}+t_{i}(n-1,0)}{\sum_{j=0}^{n-1} \eta_{j+1} e^{-S_{j}}(1+d(n-1, j))+e^{-S_{n}}}, \quad i=1, \ldots, p .
$$

Учитывая очевидное неравенство

$$
1 \geqslant \widetilde{e}_{i} F_{0}\left(F_{1} \ldots\left(F_{n-1}(0)\right) \ldots\right) \geqslant \widetilde{e}_{i} F_{0}\left(F_{1} \ldots\left(F_{m-1}(0)\right) \ldots\right), \quad m<n,
$$

получаем, что $V_{i}(n) \leqslant V_{i}(m), m<n, i=1, \ldots, p$, откуда в силу $(24),(32),(41)$ следует, что

$$
h(n) \leqslant c_{4},
$$

где $c_{4}>1 / \min _{i=1, \ldots, p} r_{i}$. Известно ([9], формула (27)), что

$$
Y(n) \leqslant 1 \text {. }
$$

Из (42). и (44) получаем, что при достаточно больших $n$

$$
|h(n)-Y(n)| \leqslant c_{4} \sum_{i=0}^{n-1} \eta_{i+1} e^{-S_{i}} \varphi(n-i)
$$

где функция $\varphi(n)$ определена в (31). Покажем, что

$$
\underset{n \rightarrow \infty}{\limsup } M_{n} \sum_{i=0}^{n-1} \eta_{i+1} e^{-S_{i}} \varphi(n-i)=0 .
$$


Для каждого фиксированного $0<\delta<1 / 4$

$$
\begin{aligned}
\sum_{i=0}^{n-1} \eta_{i+1} e^{-S_{i}} \varphi(n-i)=\sum_{i=0}^{[n / 2]} \eta_{i+1} e^{-S_{i}} \varphi(n-i) & +\sum_{i=[n / 2]+1}^{[(1-\delta) n]} \eta_{i+1} e^{-S_{i}} \varphi(n-i) \\
& +\sum_{i=[(1-\delta) n]+1}^{n-1} \eta_{i+1} e^{-S_{i}} \varphi(n-i)
\end{aligned}
$$

Из ([9], формулы (35), (13)) следует, что

$$
\mathbf{M}_{n} \sum_{i=0}^{[n / 2]} \eta_{i+1} e^{-S_{i}} \leqslant c_{5} \int_{0}^{\infty} e^{-x}\left(\mathbf{M} \eta_{1}(x+1)+\mathbf{M}\left|X_{1} \eta_{1}\right|\right) d U(x)
$$

где

$$
U(x)=\sum_{i=0}^{\infty} U_{i}(x), \quad U_{i}(x)=\mathbf{P}\left(S_{j} \leqslant S_{i}, 0 \leqslant j<i, S_{i} \leqslant x\right), \quad c_{5}>0 .
$$

Применяя к $U(x)$ теорему восстановления, получаем, что правая часть (48) не превосходит некоторой константы $c_{6}>0$. Поэтому, ввиду (31),

$$
\mathbf{M}_{n} \sum_{i=0}^{[n / 2]} \eta_{i+1} e^{-S_{i}} \varphi(n-i) \leqslant c_{7} \beta^{[n / 2]} \rightarrow 0
$$

где $c_{7}-$ положительная постоянная, и при $n \rightarrow \infty$ правая часть стремится к нулю.

Из доказательства формулы (36) в [9] следует, что

$$
\underset{n \rightarrow \infty}{\limsup } \mathbf{M}_{n}\left(\sum_{i=[n / 2]+1}^{[(1-\delta) n]} \eta_{i+1} e^{-S_{i}}\right)=0
$$

В работе ([8], стр. 231) показано, что

$$
\mathbf{M}_{n} \sum_{i=[(1-\delta) n]+1}^{n-1} \eta_{i+1} e^{-S_{i}} \leqslant c_{8} \delta, \quad c_{\delta}>0
$$

Так как $\delta>0$ можно взять сколь угодно малым, то из (31), (47), (49)-(51) получаем (46). Из (36), (40), (45), (46) следует утверждение леммы.

Замечание. Аналогичными рассуждениями, используя соотношение ([9], лемма 2)

$$
\lim _{n \rightarrow \infty} \mathbf{M}_{n} Y(n, a, b)=\widehat{\mathbf{M}}\left(\left(a+b \sum_{i=0}^{\infty} \eta_{i+1} e^{-S_{i}}\right)^{-1}\right)
$$

можно показать, что для любых $a \geqslant 0, b \geqslant 1, v>0, l>0, c>0$

$\lim _{n \rightarrow \infty} \mathbf{M}_{n} h^{+}(n, a, b, v, l, c)=\lim _{n \rightarrow \infty} \mathbf{M}_{n} h_{-}(n, a, b, v, l, c)=\widehat{\mathbf{M}}\left(\left(a+b \sum_{i=0}^{\infty} \eta_{i+1} e^{-S_{i}}\right)^{-1}\right)$. 
Известно ([9], формула (38)), что при $n \rightarrow \infty$ для любого фиксированного $j=$ $0,1, \ldots$

$$
\mathrm{M}\left(Y(n) ; \tau_{j} \leqslant n, \tau_{j+1}>n\right) \sim c_{3} A_{j} / \sqrt{n}
$$

где

$$
A_{j}>0, \quad j=0,1, \ldots, \quad \sum_{j=0}^{\infty} A_{j}<\infty .
$$

Покажем, что для величин $h(n)$ имеет место аналогичное утверждение.

Лемма 3. При каждом фиксированном $j \geqslant 1, n p u n \rightarrow \infty$

$$
\mathbf{M}\left(h(n) ; \tau_{j} \leqslant n, \tau_{j+1}>n\right) \sim \frac{c_{3} A_{j}}{\sqrt{n}} .
$$

Доказателъство. Воспользуемся методом, предложенным в работе [9]. Зафиксируем $0<\delta<1$. Известно ([9], формула (20)), что при $n \rightarrow \infty$

$$
\mathbf{P}\left(\tau_{j} \leqslant n, \tau_{j+1}>n\right)-\mathbf{P}\left(\tau_{j} \leqslant \delta n, \tau_{j+1}>n\right)=o(1 / \sqrt{n}) .
$$

Рассмотрим

$$
\mathrm{M}\left(h(n) ; \tau_{j} \leqslant \delta n, \tau_{j+1}>n\right) .
$$

Так как

$$
\left\{\tau_{j} \leqslant \delta n, \tau_{j+1}-\tau_{j}>n\right\} \subseteq\left\{\tau_{j} \leqslant \delta n, \tau_{j+1}>n\right\} \subseteq\left\{\tau_{j} \leqslant \delta n, \tau_{j+1}-\tau_{j}>(1-\delta) n\right\}
$$

To

$$
\begin{aligned}
\mathrm{M}\left(h(n) ; \tau_{j} \leqslant \delta n, \tau_{j+1}-\tau_{j}>n\right) & \leqslant \mathbf{M}\left(h(n) ; \tau_{j} \leqslant \delta n, \tau_{j+1}>n\right) \\
& \leqslant \mathbf{M}\left(h(n) ; \tau_{j} \leqslant \delta n, \tau_{j+1}-\tau_{j}>(1-\delta) n\right) .
\end{aligned}
$$

Ясно, что при $\tau_{j}<n$

$$
\begin{aligned}
\sum_{i=0}^{n-1} \eta_{i+1} e^{-S_{i}}(1+ & d(n-1, i))+e^{-S_{n}}=\sum_{i=0}^{\tau_{j}-1} \eta_{i+1} e^{-S_{i}}(1+d(n-1, i)) \\
& +e^{-S_{\tau_{j}}}\left(\sum_{i=\tau_{j}}^{n-1} \eta_{i+1} e^{-\left(S_{i}-S_{\tau_{j}}\right)}(1+d(n-1, i))+e^{-\left(S_{n}-S_{\tau_{j}}\right)}\right)
\end{aligned}
$$

Положим $\eta_{i}^{\prime}=\eta_{\tau_{j}+i}, S_{i}^{\prime}=S_{\tau_{j}+i}-S_{\tau_{j}}, \tau_{1}^{\prime}=\tau_{j+1}-\tau_{j}^{\prime}, d^{\prime}(k, l)=d\left(\tau_{j}+k, \tau_{j}+l\right)$. Из $(24),(28)$, и (42) следует, что существует такое $m^{*}$, что для всех $n>n^{\prime}>m>m^{*}$

$$
\left(1+c_{9} \varphi(m)\right)^{-1} h^{-1}(m) \leqslant h^{-1}\left(n^{\prime}\right) \leqslant h^{-1}(n)\left(1+c_{9} \varphi(m)\right),
$$

где $c_{9}>0$. Для $\tau_{j} \leqslant \delta n$ выражение в скобках в (59) при достаточно больших $n$ согласно (60) ограничено сверху и снизу, соответственно, величинами

$$
\left(1+c_{9} \varphi([(1-\delta) n])\right)\left(\sum_{i=0}^{n-1} \eta_{i+1}^{\prime} e^{-S_{i}^{\prime}}\left(1+d^{\prime}(n-1, i)\right)+e^{-S_{n}^{\prime}}\right)
$$


и

$$
\frac{1}{1+c_{9} \varphi([(1-\delta) n])}\left(\sum_{i=0}^{[(1-\delta) n]-1} \eta_{i+1}^{\prime} e^{-S_{i}^{\prime}}\left(1+d^{\prime}([(1-\delta) n]-1, i)\right)+e^{-S_{[(1-\delta) n]}^{\prime}}\right) .
$$

Исследуем верхнюю оценку для выражения (57), которая при достаточно больших $n$ согласно (30), (58), (59) и (62) может быть записана в виде

$$
\begin{aligned}
& \mathbf{M}\left(\left(\sum_{i=0}^{\tau_{j}-1} \eta_{i+1} e^{-S_{i}}(1-\varphi([(1-\delta) n]))\right.\right. \\
& \quad+\left(1+c_{9} \varphi([(1-\delta) n])\right)^{-1} e^{-S_{\tau_{j}}}\left(\sum _ { i = 0 } ^ { [ ( 1 - \delta ) n ] - 1 } \eta _ { i + 1 } ^ { \prime } e ^ { - S _ { i } ^ { \prime } } \left(1+d^{\prime}([(1-\delta) n]-1, i)\right.\right. \\
& \left.\left.\left.\quad+e^{-S_{[(1-\delta) n]}^{\prime}}\right)\right)^{-1} ; \tau_{1}^{\prime}>(1-\delta) n\right) .
\end{aligned}
$$

Так как последовательность $\left\{\eta_{i}^{\prime}, d_{i}^{\prime}, S_{i}^{\prime}\right\}$ есть точная вероятностная копия исходной последовательности $\left\{\eta_{i}, d_{i}, S_{i}\right\}$ и не зависит от $S_{1}, \ldots, S_{\tau_{j}}$, то случайные величины $\sum_{i=0}^{\tau_{j}-1} \eta_{i+1} e^{-S_{i}}, e^{-S_{\tau_{j}}}$ не зависят от случайной величины

$$
\sum_{i=0}^{[(1-\delta) n]-1} \eta_{i+1}^{\prime} e^{-S_{i}^{\prime}}\left(1+d^{\prime}([(1-\delta) n-1, i))+e^{-S_{[(1-\delta) n]}^{\prime}}\right.
$$

а последняя имеет такое же распределение, как если бы штрихи над $\eta_{i}, S_{i}, d_{i}$ отсутствовали. Будем обозначать $\mathbf{M}_{n}^{\prime}$ условное математическое ожидание при условии величин $\eta_{1}, \ldots, \eta_{\tau_{j}}, S_{1}, \ldots, S_{\tau_{j}}, \tau_{j}$ и события $\left(\tau_{1}^{\prime}>(1-\delta) n\right)$. Запишем $(63)$ в виде

$$
\begin{aligned}
& \mathbf{M}\left(\mathbf { M } _ { n } ^ { \prime } \left(\sum_{i=0}^{\tau_{j}-1} \eta_{i+1} e^{-S_{i}}(1-\varphi([n(1-\delta)]))\right.\right. \\
&+\left(1+c_{9} \varphi([(1-\delta) n])\right)^{-1} e^{-S_{\tau_{j}}}\left(\sum_{i=0}^{[(1-\delta) n]-1} \eta_{i+1}^{\prime} e^{-S_{i}^{\prime}}\left(1+d^{\prime}([(1-\delta) n]-1, i)\right)\right. \\
&\left.\left.\left.+e^{-S_{[(1-\delta) n]}^{\prime}}\right)\right)^{-1}\right) \mathbf{P}\left(\tau_{1}^{\prime}>(1-\delta) n\right) .
\end{aligned}
$$

При $n \rightarrow \infty$ согласно (53) внутреннее математическое ожидание в (64) сходится с вероятностью единица $\mathrm{K}$

$$
\widehat{\mathbf{M}}^{\prime}\left(\sum_{i=0}^{\tau_{j}-1} \eta_{i+1} e^{-S_{i}}+e^{-S_{\tau_{j}}} \sum_{i=0}^{\infty} \eta_{i+1}^{\prime} e^{-S_{i}^{\prime}}\right)^{-1}
$$

где символ $\widehat{\mathbf{M}}^{\prime}$ означает, что математическое ожидание вычисляется при фиксированных значениях $\eta_{1}, \ldots, \eta_{\tau_{j}}, \tau_{j}, S_{1}, \ldots, S_{\tau_{j}}$, а распределение процесса $\left(S_{i}^{\prime}, \eta_{i}^{\prime}\right), i=$ 
$1,2, \ldots$, соответствует мере $\widehat{\mathbf{P}}$. Так как в силу (43) подынтегральная функция в (64) ограничена, применяя теорему Лебега о предельном переходе под знаком интеграла, получаем, что

$$
\limsup _{n \rightarrow \infty}\left\{\sqrt{n} \mathbf{M}\left(h(n) ; \tau_{j} \leqslant \delta n, \tau_{j+1}-\tau_{j}>(1-\delta) n\right)\right\} \leqslant \frac{c_{3} A_{j}}{\sqrt{1-\delta}},
$$

где

$$
A_{j}=\mathbf{M} \widehat{\mathbf{M}}^{\prime}\left(\sum_{i=0}^{\tau_{j}-1} \eta_{i+1} e^{-S_{i}}+e^{-S_{\tau_{j}}} \sum_{i=0}^{\infty} \eta_{i+1}^{\prime} e^{-S_{i+1}^{\prime}}\right)^{-1}
$$

Аналогично получаем, что

$$
\liminf _{n \rightarrow \infty}\left\{\sqrt{n} \mathrm{M}\left(h(n) ; \tau_{j} \leqslant \delta n, \tau_{j+1}-\tau_{j}>n\right)\right\} \leqslant c_{3} A_{j}
$$

Из (56), (66), (68) следует утверждение леммы.

Напомним, что в силу (32), (41)

$$
V_{i}(n)=\left(r_{i}+t_{i}(n-1,0)\right) h(n), \quad i=1, \ldots, p .
$$

Из лемм 2,3 и соотношения (28) следует, что при $n \rightarrow \infty$ для любого фиксированного $j=0,1, \ldots$

$$
\mathrm{M}\left(V_{i}(n) ; \tau_{j} \leqslant n, \tau_{j+1}>n\right) \sim \frac{c_{3} r_{i} A_{j}}{\sqrt{n}} .
$$

Лемма 4. Имеет место соотношение

$$
\lim _{m \rightarrow \infty} \limsup _{n \rightarrow \infty} \sqrt{n} \mathbf{M}\left(V_{i}(n) ; \tau_{m} \leqslant n\right)=0 .
$$

Доказателъство. Из (43) и (59) следует, что

$$
\begin{aligned}
\mathbf{M}\left(V_{i}(n) ; \tau_{m} \leqslant n\right) & =\sum_{j=m}^{n} \mathbf{M}\left(V_{i}(n) ; \tau_{j} \leqslant n, \tau_{j+1}>n\right) \\
& \leqslant \sum_{j=m}^{n} \sum_{k=m}^{n} \mathbf{M}\left(V_{i}(n) ; \tau_{j}=k, \tau_{j+1}-\tau_{j}>n-k\right) \\
& \leqslant c_{10} \sum_{k=m}^{n} \mathbf{P}\left(\tau_{1}>n-k\right) \sum_{j=m}^{k} \mathbf{M}\left(e^{S_{\tau_{j}}} ; \tau_{j}=k\right), \quad c_{10}>0 .
\end{aligned}
$$

Известно ([12], формулы (5.12), (5.13)), что для произвольного $j \in \mathbf{N}$ и достаточно больших $n$

$$
\mathbf{M}\left(e^{S_{\tau_{j}}} ; \tau_{j}=n\right) \leqslant c_{11} \lambda^{j} j n^{-3 / 2},
$$

где $c_{11}>0,0<\lambda<1$. Из (73) получаем, что при достаточно больших $m$

$$
\sum_{j=m}^{k} \mathbf{M}\left(e^{S_{\tau_{j}}} ; \tau_{j}=k\right) \leqslant \varepsilon(m) k^{-3 / 2}
$$


где $\varepsilon(m) \rightarrow 0$ при $m \rightarrow \varepsilon$.

Из (36) следует, что при $n \geqslant 1$

$$
\mathbf{P}\left(\tau_{1}>n\right)<c_{12} n^{-1 / 2},
$$

где $c_{12}>0$. Несложно показать, что

$$
\lim _{m \rightarrow \infty} \varepsilon(m) \lim _{n \rightarrow \infty} \sqrt{n} \sum_{k=m}^{n-1}(n-k)^{-1 / 2} k^{-3 / 2}=0 .
$$

Соотношение (71) вытекает из (72)-(76).

Остается заметить, что из (54), (70), (71) следует утверждение теоремы, причем

$$
d_{i}=c_{3} r_{i} \sum_{j=0}^{\infty} A_{j}
$$

Докажем теперь теорему 1 . Обозначим $\varkappa_{n}=\max \left(-S_{0},-S_{1}, \ldots,-S_{n}\right)$. Известно ([9], с. 815), что если $\mathbf{M} X_{1}=0,0<\mathbf{M} X_{1}^{2}<\infty$, то при $n \rightarrow \infty$

$$
\mathrm{M} e^{\varkappa_{n}} \sim C_{2} / \sqrt{n}
$$

где $C_{2}-$ положительная постоянная. Ясно, что при $m<n$

$$
\tilde{e}_{j}\left(I-F_{0}\left(F_{1}\left(\ldots F_{n-1}(0) \ldots\right)\right)\right) \leqslant \widetilde{e}_{j} M_{0} \ldots M_{m} I=\prod_{k=0}^{m} \rho_{k} \widetilde{e}_{j} \prod_{i=0}^{m} \frac{M_{i}}{\rho_{i}} I, \quad j=1, \ldots, p .
$$

В силу (17), для некоторой положительной постоянной $C_{3}$

$$
\tilde{e}_{j} \prod_{i=0}^{m} \frac{M_{i}}{\rho_{i}} I<C_{3}, \quad j=1, \ldots, p .
$$

Из (79), (80) получаем, что

$$
M \widetilde{e}_{j}\left(I-F_{0}\left(F_{1}\left(\ldots F_{n-1}(0) \ldots\right)\right)\right) \leqslant C_{3} M e^{-\varkappa_{n}}, . \quad j=1, \ldots, p .
$$

Отсюда, используя (78), получаем правое неравенство в выражении (10). Чтобы получить оценку слева в соотношении (10), используем следующую оценку для многомерной производящей функции $f(s), s=\left(s_{1}, \ldots, s_{p}\right)^{T}([11]$, формулы $(64),(65))$ :

$$
f(s) \leqslant 1-N_{1}^{2} / N_{2},
$$

где

$$
N_{1}=\sum_{i=1}^{p}\left(1-s_{i}\right) \frac{\partial f}{\partial s_{i}}(I), \quad N_{2}=N_{1}+\sum_{i, j=1}^{p}\left(1-s_{i}\right)\left(1-s_{j}\right) \frac{\partial^{2} f}{\partial s_{i} \partial s_{j}}(I) .
$$

Таким образом, (81) можно переписать в виде

$$
f(s) \leqslant 1-\sum_{i=1}^{p} \frac{\partial f}{\partial s_{i}}(I)\left(1-s_{i}\right)\left(1+\frac{\sum_{i, j=1}^{p}\left(1-s_{i}\right)\left(1-s_{j}\right) \frac{\partial^{2} f}{\partial s_{i} \partial s_{j}}(I)}{\sum_{i, j=1}^{p}\left(1-s_{i}\right) \frac{\partial f}{\partial s_{i}}(I)}\right)^{-1} .
$$


Рассмотрим $F^{(\theta)}(s)=\left(F_{1}^{(\theta)}(s), \ldots, F_{p}^{(\theta)}(s)\right)$, удовлетворяющую условиям теоремы 1. Положим

$$
G^{(\theta)}(s)=I-\frac{M^{(\theta)}(I-s)}{1+\widetilde{\Gamma}(\theta)(I-s)},
$$

где $M^{(\theta)}$ - матрица математических ожиданий для $F^{(\theta)}(s)$, а $p$-мерный векторстрока $\widetilde{\Gamma}(\theta)=\left(\Gamma_{1}(\theta), \ldots, \Gamma_{p}(\theta)\right)$ имеет одинаковые компоненты $\Gamma_{j}(\theta)=\Gamma, j=$ $1, \ldots, p$, где

$$
\Gamma=\max \left\{\frac{D_{2}}{m}, \frac{p D_{2}}{K}\right\},
$$

$m, D_{2}$ - постоянные из условий $(5),(6), K=\min \{p,|r|\}$. Ясно, что $G^{(\theta)}(s) \in$ $H\left(m, D_{1}, D_{2}, D_{3}\right)$, где $D_{3}=2 \Gamma D_{2}$. Если в качестве $f(s)$ рассмотреть $F_{i}^{(\theta)}(s), \theta \in \Theta$, $i=1, \ldots, p$, то из (82)-(84) легко получить, что

$$
F_{i}^{(\theta)}(s) \leqslant G_{i}^{(\theta)}(s), \quad \theta \in \Theta, \quad i=1, \ldots, p,
$$

где $G_{i}^{(\theta)}(s)-i$-я компонента вектора $G^{(\theta)}(s)$.

Положим

$$
G_{n}(s)=G^{\left(\zeta_{n}\right)}(s)
$$

Таким образом,

$$
\mathbf{M} \widetilde{e}_{i}\left(I-G_{0}\left(G_{1}\left(\ldots\left(G_{n-1}(0)\right) \ldots\right)\right)\right) \leqslant \mathbf{M} \widetilde{e}_{i}\left(I-F_{0}\left(F_{1}\left(\ldots\left(F_{n-1}(0)\right) \ldots\right)\right)\right) .
$$

Применяя теорему 2 к левой части в (86) и используя (3), получаем нижнюю оценку в (10). Теорема доказана.

Автор выражает искреннюю благодарность рецензенту за ряд замечаний, существенно способствовавших улучшению работы.

\section{Список литературы}

1. Smith W. L., Wilkinson W. On branching processes in random environment. Ann. Math. Statist. (1969) 40, №3, 814-827.

2. Athreya K. B., Karlin S. On branching processes with random environments I, II. Ann. Math. Statist. (1971) 42, №5, 1499-1520; (1971) 42, №6, 1843-1858.

3. Agresti A. Bounds on the extinction time distribution of a branching process. Adv. Appl. Prob. (1974) 6, №2, 322-335.

4. Agresti A. On the extinction times of varying and random environment branching processes. J. Appl. Prob. (1975) 12, №1, 39-46.

5. Tanny D. On multitype branching processes in a random environment. Adv. Appl. Prob. (1981) 13, №3, 464-497.

6. Weissener E. W. Multitype branching processes in random environments. J. Appl. Prob. (1971) 8, №1, 17-31.

7. Kaplan N. Some results about multidimensional branching processes with random environments. Ann. Prob. (1974) 2, №3, 441-455. 
8. Borovkov K. A., Vatutin V. A. Reduced critical branching processes in random environment. Stochastic Processes and their Applications (1997) 71, №2, 225-240.

9. Козлов М. В. Об асимптотике вероятности невырождения критических ветвящихся процессов в случайной среде. Теория вероятн. и ее примен. (1976) 21, №4, 813-825.

10. Fleischmann K., Vatutin V. A. Reduced subcritical Galton-Watson processes in a random environment. Preprint No. 306 of the Weierstraß-Institut für Angewandte Analysis und Stochastik, Berlin, 1996.

11. Зубков А. М. Неравенства для распределения числа одновременно происходящих событий. Обозрение прикладной и промыиленной математики (1994) 1, №4, 638-666.

12. Ватутин В. А.; Дьяконова Е. Е. Критические ветвящиеся процессы в случайной среде: вероятности вырождения в фиксированный момент. Дискретная математика (1997) 9, №4, 100-126.

13. Athreya K. B., Ney P. E. Branching Processes. Springer, Berlin, 1972.

14. Гантмахер Ф. Р. Теория матриц. Наука, Москва, 1988.

15. Kesten H., Spitzer F. Convergence in distribution of products of random matrices. Z. Wahrsch. verw. Geb. (1984) 67, №4, 363-386.

16. Феллер В. Введение в теорию вероятностей и ее приложения, т.2. Мир, Москва, 1984.

Статья поступила 29.12.97. Переработанный вариант поступил 14.05.98. 